\title{
QSO EMISSION LINE REDSHIFT DIFFERENCES
}

\author{
Vesa Junkkarinen \\ The Center for Astrophysics and Space Sciences C-011 \\ The University of California, San Diego \\ La Jolla, CA 92093
}

\section{INTRODUCTION}

Redshift differences between QSO emission lines reflect the kinematical differences between the regions where the lines are produced. Gaskell (1982) discovered a systematic redshift difference between the broad lines of high ionization and the narrow forbidden lines that amounted to a blueshifting of C IV $\lambda 1549$ by 600 $\mathrm{km} \mathrm{s}^{-1}$. Gaskell obtained this result basically by comparing C IV emission and Mg II $\lambda 2798$ emission in one sample of QSOs and by comparing Mg II emission and the narrow forbidden lines in a different sample. So the distribution of C IV emission redshifts relative to the narrow forbidden lines was not sampled directly by Gaskell. By observing QSOs with redshifts near 1.3, it is possible to directly sample the redshift difference between $\mathrm{C}$ IV and the narrow forbidden lines. This redshift difference and its distribution should provide kinematical information on the clouds of gas that give rise to the broad lines of high ionization. The narrow forbidden lines are the preferred velocity reference because they may reflect the systemic velocity of the QSO.

\section{OBSERVATIONS AND RESULTS}

Redshift 1.3 QSOs were selected in order to observe C IV $\lambda 1549$ emission from ground based telescopes and to observe, in the same QSOs with currently available CCD equipped spectrographs, [O II] $\lambda 3727$ redshifted to the red spectral region. Observations have been obtained at KPNO and at Lick Observatory. The spectral resolutions used are $400-600 \mathrm{~km} \mathrm{~s}^{-1} \mathrm{FWHM}$ in velocity units. With these spectra, redshift differences can usually be measured to an accuracy of better than $200 \mathrm{~km}$ $\mathrm{s}^{-1}$ although a few of the broad C IV $\lambda 1549$ emission lines have line profiles that are not very peaked and may have errors a few times this amount. To determine the redshifts, the wavelengths of the emission lines are measured by taking the first moment of the flux above the $50 \%$ fractional intensity level referenced to the peak intensity in the emission line.

Of the sample of 35 QSOs with spectra in the red spectral region, 13 QSOs have narrow forbidden lines strong enough to be detected and measured. The median redshift difference between the C IV $\lambda 1549$ line and the narrow forbidden lines is $+30 \mathrm{~km} \mathrm{~s}^{-1}$ in velocity units with the sign convention that positive values indicate that $\mathrm{C}$ IV is redshifted with respect to the narrow forbidden lines. So 
the $-600 \mathrm{~km} \mathrm{~s}^{-1}$ redshift difference found by Gaskell (1982) is not confirmed by this sample. The distribution of redshift differences is consistent with a Gaussian distribution with a mean of $+40 \mathrm{~km} \mathrm{~s}^{-1}$ and $\sigma=230 \mathrm{~km} \mathrm{~s}^{-1}$. So the formal error in the mean, assuming that the true distribution is Gaussian, is $70 \mathrm{~km} \mathrm{~s}^{-1}$ (one $\sigma$ ). For this sample, C IV $\lambda 1549$ emission is at the same redshift as the narrow forbidden lines with a distribution not much broader than the width expected from the measurement errors alone.

The results of measurements of C IV and $\mathrm{Mg}$ II $\lambda 2798$ broad emission are summarized in Table I. Median and mean redshift differences in velocity units and the $\sigma$ per object assuming a Gaussian distribution in $\Delta z$ are given. Table I shows that $\mathrm{Mg}$ II $\lambda 2798$ is redshifted by roughly $400 \mathrm{~km} \mathrm{~s}^{-1}$ relative to both the narrow forbidden lines and C IV $\lambda 1549$.

\section{TABLE I}

\begin{tabular}{lcccc}
\hline $\begin{array}{l}\text { Emission Lines } \\
\text { Compared }\end{array}$ & $\begin{array}{r}\text { Number of } \\
\text { QSOs }\end{array}$ & $\begin{array}{r}\text { median } \Delta z \\
\mathrm{~km} \mathrm{~s}^{-1}\end{array}$ & $\begin{array}{r}\text { mean } \Delta z \\
\mathrm{~km} \mathrm{~s}^{-1}\end{array}$ & $\begin{array}{c}\sigma \\
\mathrm{km} \mathrm{s}^{-1}\end{array}$ \\
\hline C IV - [O II] & 13 & 30 & 40 & 230 \\
Mg II - [O II] & 12 & 420 & 380 & 270 \\
Mg II - C IV & 15 & 330 & 460 & 410 \\
\hline
\end{tabular}

The pattern of redshift differences shown by this sample of QSOs fits the qualitative predictions of some models for the dynamics of the broad emission line (BEL) clouds in QSOs. A recent model proposed by Mathews (1986) incorporates radial acceleration of BEL clouds by a combination of radiation and dynamical pressure. In this model, the optically thin BEL clouds emit C IV $\lambda 1549$ and Ly $\alpha$ while the optically thick clouds emit most of the emission observed in $\mathrm{Mg}$ II $\lambda 2798, \mathrm{Fe}$ II lines, and Balmer lines. If the optical depths are large, the faces of the optically thick clouds illuminated by the central source will be brighter resulting in a slight redshift when the BEL cloud motion is radial and outward.

The pattern of QSO emission line redshift differences is probably more complicated than that given by this sample of 13 QSOs because there are a few QSOs with $z_{a}>z_{e}$ that have C IV emission that is blue shifted relative to the narrow forbidden lines by greater than $1000 \mathrm{~km} \mathrm{~s}^{-1}$ (Junkkarinen, 1988 in preparation). Such large velocity differences would not be expected if the true distribution is a Gaussian with $\sigma=230 \mathrm{~km} \mathrm{~s}^{-1}$. Also the emission line profiles contain more information than a single wavelength. The C IV emission lines measured from this sample are relatively symmetric from the peak intensity to $50 \%$ of peak intensity, but most of the C IV lines have extended red or blue emission at smaller fractional intensity levels. These line "wings" in several cases carry a substantial fraction of the total line intensity and the first moment of the total line is then displaced by hundreds of $\mathrm{km} \mathrm{s}^{-1}$ relative the first moment taken above the $50 \%$ intensity level.

\section{REFERENCES}

Gaskell, C. M. 1982, Ap. J., 263, 79.

Mathews, W. G. 1986, Ap.J., 305, 187. 